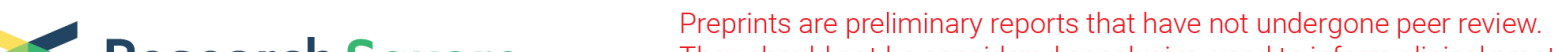 Research Square They should not be considered conclusive, used to inform clinical practice, or referenced by the media as validated information.
}

\section{Clinical Characteristics and Economic Analysis of Intramedullary Nailing Versus Plate and Screws for Open Distal Tibial Fractures: a Retrospective Study}

\section{Yibo Xu}

Department of Orthopedics Trauma, Xi'an Honghui Hospital, School of Medicine Xi'an Jiaotong University

\section{Cheng Ren}

Department of Orthopedics Trauma, Xi'an Honghui Hospital, School of Medicine Xi'an Jiaotong University

\section{Zhong Li ( $\nabla$ lizhong0607@126.com )}

\section{Kun Zhang}

Department of Orthopedics Trauma, Xi'an Honghui Hospital, School of Medicine Xi'an Jiaotong University

\section{Teng Ma}

Department of Orthopedics Trauma, Xi'an Honghui Hospital, School of Medicine Xi'an Jiaotong University

\section{Deyin Liu}

Department of Orthopedics Trauma, Xi'an Honghui Hospital, School of Medicine Xi'an Jiaotong University

\section{Yao Lu}

Department of Orthopedics Trauma, Xi'an Honghui Hospital, School of Medicine Xi'an Jiaotong University

\section{Ming Li}

Department of Orthopedics Trauma, Xi'an Honghui Hospital,School of Medicine Xi'an Jiaotong University

\section{Hongliang Liu}

Department of Orthopedics Trauma, Xi'an Honghui Hospital, School of Medicine Xi'an Jiaotong University

\section{Wang Tao}

Department of Orthopedics Trauma, Xi'an Honghui Hospital, School of Medicine Xi'an Jiaotong University

\section{Ning Duan}

Department of Orthopedics Trauma, Xi'an Honghui Hospital, School of Medicine Xi'an Jiaotong University 


\section{Ning Jiao}

Department of Orthopedics Trauma, Xi'an Honghui Hospital, School of Medicine Xi'an Jiaotong University

\section{Daigang Lu}

Department of Orthopedics Trauma, Xi'an Honghui Hospital, School of Medicine Xi'an Jiaotong University

\section{Qian Wang}

Department of Orthopedics Trauma, Xi'an Honghui Hospital, School of Medicine Xi'an Jiaotong University

\section{Hanzhong Xue}

Department of Orthopedics Trauma, Xi'an Honghui Hospital, School of Medicine Xi'an Jiaotong University

\section{Research article}

Keywords: economic analysis, intramedullary nailing, locking compression plate, open fracture, distal tibial fractures

Posted Date: June 19th, 2020

DOI: https://doi.org/10.21203/rs.3.rs-36128/v1

License: (c) (1) This work is licensed under a Creative Commons Attribution 4.0 International License. Read Full License 


\section{Abstract}

Background To analyze the clinical characteristic and economic of intramedullary nailing versus locking compression plate for the treatment of open distal tibial fractures.

Methods A retrospective analysis was conducted by enrolling patients with open distal tibial fractures who were admitted in Lower Limb Surgery Ward of Traumatic Orthopedic Department $₫$ X'an Honghui Hospital from January 2016 to January 2019. The collected clinical materials and data included gender, age, injury mechanism, smoke and alcohol, comorbidity disease, Gustilo classification, days before operation, final treatment option, surgical duration, intraoperative bleeding loss, length of stay, complications, Johner-Wruhs criteria, total cost within 1 year after surgery (including hospitalization expenses and postoperative rehabilitation or follow-up expenses). All the patients were divided into two groups based on the final treatment options: Group IMN(intramedullary nailing) and Group LCP(locking compression plate). Cost data for each case within 1 year after surgery were analyzed for the costeffectiveness ratio (CER)and incremental cost-effectiveness ratio (ICER) of IMN versus LCP.

Results 49 consecutive cases were enrolled including 28 cases of Group IMN and 21 cases of Group LCP with the excellent and good rate of $82.1 \%$ ( 23 cases) and $85.7 \%$ (18 cases) respectively based on JohnerWruhs criteria. The surgical duration $(p=0.017)$ and intraoperative bleeding loss $(p=0.046)$ were significantly lower in Group IMN than in Group LCP. Total cost within 1 year after surgery $(\mathrm{p}=0.048)$ was

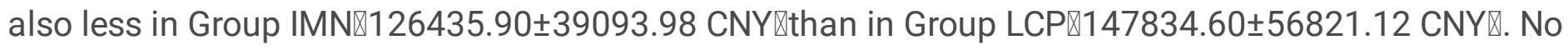
statistically significant difference was discovered on the excellent and good rate of Johner-Wruhs criteria between the two groups(p囚0.05). The average cost for every $1 \%$ of excellent and good rate was 1540.02 CNY in Group IMN and 1725.02 CNY in Group LCP. Each 1\% increasing of excellent and good rate cost 5944.08 CNY more in Group LCP compared with Group IMN.

Conclusions Both the IMN and LCP could provide a satisfactory outcome for open distal tibial fractures. However the IMN was a more cost-effective management than LCP when the economic analysis was included.

\section{Introduction}

Open fractures always result from high-energy injury and severe one is often associated with unpredictable complications and disabilities which could lead to a significant higher long-term morbidity and mortality even treated and managed by sophisticated orthopedics trauma surgeon [1-3]. High energy injury including road traffic accidents, falls from a standing height and crush injury is the most common cause $(20 \%)$ for open fractures, meanwhile the fractures of lower extremity are the first most common open fractures with an incidence of $22 \%$ in all open shaft fractures [4, 5].

The final management option after early surgical debridement and fracture stabilization for open tibial fractures depends on extend of injury, fracture location and pattern, severity of soft tissue injury, general hemodynamic status and the experience of surgeon. The most common options include external fixation, 
intramedullary nailing and internal fixation with plate and screws [6]. The plates are frequently used for fractures extended to the joints and nails are for extra-articular fractures. Moreover, currently IMNs are also used for fractures with a limited extension to the joint and indications for their use is extending as they are better choices particularly for open fractures [1,4-6]. Complex open distal tibial fractures are always associated with severe soft tissue injury, sometimes even with the onset of segmental bone defect. Hence the treatment of this limb threatening injury faces a substantial challenge of more accurate clinical decision making, longer treatment time span and increased financial burden for both patients and social insurance system.

The aim of this article is to assess the clinical characteristic and total costs for patients with open distal tibial fractures treated in our trauma center, specifically to analyze the cost-effectiveness ratio and incremental cost-effectiveness ratio of intramedullary nailing versus locking compression plate for the treatment of open distal tibial fractures.

\section{Materials And Methods}

\section{Study design}

The inclusion criteria were patients aged over 14 years old, diagnosed as open distal tibial fractures, AO classification as type 43-A, no vascular or nerve injuries, final implant option is intramedullary nailing or plate with screws, complete clinical demographic and radiographic data, the exclusion criteria were pathological fractures, polytrauma patients and incomplete clinical demographic and radiographic data.

A single-center, case control, retrospective study was conducted at Lower Limb Surgery Ward of Traumatic Orthopedic Department $₫ X i ' a n$ Honghui Hospital from January 2016 to January 2019. All eligible data was collected and reviewed based on the system included; age, gender, mechanism of injury, history of smoke and alcohol, comorbidity disease, AO classification, Gustilo classification, mangled extremity severity score(MESS), days before operation, final treatment option, surgical duration, intraoperative bleeding loss, length of stay, complications, Johner-Wruhs criteria[7], total cost within 1 year after surgery (including hospitalization expenses and postoperative rehabilitation or follow-up expenses).

All the patients were divided into two groups based on the final treatment options: Group IMN and Group LCP. Cost data for each case within 1 year after surgery were analyzed for the cost-effectiveness ratio (CER)and incremental cost-effectiveness ratio (ICER) of IMN versus LCP. In our study we used the CER as the average cost/ the excellent and good rate of Johner-Wruhs criteria* 100 , meaning the average cost for every $1 \%$ of excellent and good rate in each group. The ICER was the difference of average cost between the two groups/ the difference of excellent and good rate between the two groups ${ }^{\star} 100$, meaning how much did it cost more for each $1 \%$ increasing of excellent and good rate in Group LCP compared with Group IMN.

\section{Surgical procedure}


Debridement surgery was performed after accomplishing the preoperative estimating by debriding all contaminated and nonviable tissue. Irrigation volume depended on the severity of fracture with $3 \mathrm{~L}$ for type I, $6 \mathrm{~L}$ for type II and $9 \mathrm{~L}$ for type III fractures [8]. External fixation was performed as a temporizing fixation and vacuum sealing drainage was used for temporary coverage between further debridement or until flap coverage. Second debridement surgery might be in progress within 24 to 48 hours. Whenever the soft tissue viability or adequacy of debridement was questionable, further debridement would be necessary to prevent infection before performing the final internal fixation [9]. The final fixation options were variable depending on the soft tissue injury, the pattern of fracture and the experience of orthopedic surgeon. When intramedullary nailing was applied for the final option (Fig.1), it was critical to differentiate and correct the rotational deformity and angular malalignment during surgery [10]. However, fractures associated with metaphysis fracture and soft tissue condition permitted the alternative option, we might refer to minimally invasive plates and screws as the final fixation (Fig.2).

\section{Statistical analysis}

SPSS24.0 was used for all data analysis and Pख0.05 selected as the threshold for statistical significant. A Pearson test for normality was conducted for all continuous data, and the continuous data with the normal distribution was described in the form of mean \pm standard deviation whereas the categorical data was described in number of cases (percentage). The Student's t-test was conducted for continuous variables with the normal distribution and the Mann-Whitney U-test for those not with the normal distribution. As for the categorical variables, the $\chi 2$ test was performed.

\section{Outcomes}

We enrolled 49 consecutive patients treated by intramedullary nail (Group IMN,28 cases) or plate (Group LCP,21 cases) as the final treatment option. The excellent and good rate were $82.1 \%$ and $85.7 \%$ respectively based on the Johner-Wruhs criteria.

\section{Demographic result}

The demographic data of the patients are shown in Table1, and there was no statistically significant difference between the two groups in terms of gender, age, history of smoke and alcohol, comorbidity disease, injury mechanism, AO classification, Gustilo classification, mangled extremity severity score (MESS). The comorbidity disease of the patients mainly included hypertension, diabetes and coronary heart disease.

\section{Perioperative result}

The perioperative period data including injury to surgery interval, times of surgical debridement, final treatment option, total surgical duration, total length of hospital stay, the onset of complications including infection(surgical site, lung and urinary system), deep vein thrombosis, bedsore, compartment syndrome, delayed union and nonunion are shown in Table 2. The total surgical duration and total 
intraoperative bleeding loss were significant lower in Group IMN than in Group LCP, meanwhile no statistically significant difference of other variables was found between the two groups.

\section{Economic analysis}

The mean total cost within 1 year after surgery of Group IMN was $(126435.90 \pm 39093.98)$ CNY and that of Group LCP was (147834.60 \pm 56821.12$)$ CNY, the cost of Group IMN was significantly lower than that of Group LCP. The principal outcome calculated was the incremental cost-effectiveness ratio (ICER), which is the ratio of the difference in costs to the difference in excellent and good rate of Johner-Wruhs criteria between the two groups in our study. The mean total cost for every $1 \%$ of excellent and good rate was 1540.02 CNY in the Group IMN, which was favored more than the Group LCP of 1725.02 CNY. Each 1\% increasing of excellent and good rate cost 5944.08 CNY more in Group LCP compared with Group IMN.

\section{Discussion}

Typically, open fractures are often associated with a higher incidence of infection, malunion and nonunion as a result of the connection between fracture site and in vitro environment, and the prognosis is always unpredictable when prompt and correct management is a substantial challenge for orthopedic surgeon [11]. An ideal treatment for severe open fractures requires the comanagement of both senior orthopedics and plastic surgeons [12].

The principal aim of treating complex open distal tibial fractures is to restore the alignment and extremity function as good as possible, which results in higher treatment demand, longer treatment time span and increased financial burden for both patients and social insurance system [13]. Thus we aim to assess the total costs for patients with open distal tibial fractures treated in our trauma center, specifically to analyze the cost-effectiveness ratio and incremental cost-effectiveness ratio of intramedullary nailing versus locking compression plate for the treatment of open distal tibial fractures (type 43-A).

We consulted the cost-effectiveness method for economic analysis which is a form of economic analysis using specific clinical treatment outcome measures (physiological parameters, functional status, qualityadjusted life-year, etc.) as indicators to compare the relative costs and outcomes (effects) of different courses of treatment, which applies economic principles, methods, and analytical techniques to evaluate the clinical treatment outcomes and guide surgeon to choose a reasonable treatment option[14]. Several studies also demonstrated its reliability for economic evaluation of specific treatment options [15]. We chose the excellent and good rate of Johner-Wruhs criteria as the indicator in this article.

There are many different final management options for open distal tibial fractures after early surgical debridement and fracture stabilization. The extent and condition of soft tissue is a prerequisite for any management options $[16,17]$. Intramedullary nail is considered the optimal management choice in most case scenario $[6,13,18,19]$, it is capable of providing early weight bearing and lower incidence of malalignment with less interference in the soft tissue of fracture site. Currently the indications for the IMN use is extending for distal tibial fractures with the improvement of nail design and application of a variety 
of the reduction techniques presented to minimize the malalignment such as poller screws and plateassistant reduction. Conventional plating techniques are not favored for open tibial fractures considering the extensive soft tissue loss. However, minimal invasive plating techniques which could provide mechanical stability and diminish the interference in the soft tissue to protect local blood supply simultaneously have emerged and make the plates and screws fixation to be a supplement option when it's associated with open metaphysis or intra-articular fracture [20]. Different treatment options result in different hospital costs.

In this article Group LCP had significantly higher total surgical duration and intraoperative bleeding loss than did Group IMN, which was decided by the unique operative techniques of the two different implant options, the mainly affecting factors for prolonged surgical duration of Group LCP were longer duration of fracture reduction and wound closure[1, 19,20]. Meanwhile the intraoperative bleeding loss was significantly higher in Group LCP than in Group IMN when a tourniquet was performed for both groups, whereas Wang et al found that the $\mathrm{Hb}$ loss, Hct loss and surgical duration were significantly lower in Group LCP than in Group IMN after taking the hidden blood loss into account[21]. We assumed that the blood loss issue depends on the surgical duration, the experience and operation skills of orthopedic surgeon. The amount of intraoperative bleeding loss increased with prolonged surgical duration, and lead to prolonged hospitalization and an increase risk of wound infection [13], which implied the increasing of hospital costs. No statistically significant difference was found in the excellent and good rate between the two groups and demonstrated that functional or anatomical reduction was obtained based on either intramedullary nail or plate with screws, which provided an optimal prerequisite for rehabilitation.

The total cost in this article included hospitalization expenses and postoperative rehabilitation or followup expenses. The total cost in Group IMN was significantly lower than that in Group LCP, whereas the cost of implant that used indicated no statistically significantly difference between the two groups, specifically the difference mainly exists in the nursing, drug and other adjuvant therapy between the two groups. We assumed that when performing plates for patients with type 43-A open fractures, more concerns about the soft tissue condition for the orthopedic surgeon arose which resulted in more therapy strategies applying to decrease the possibility of any soft tissue associated complications, thus leading to a higher cost for Group LCP. Moreover, due to treatment for comorbidity disease during perioperative period, the hospitalization expenses for patients associated with comorbidities such as diabetes, hypertension and coronary artery disease were higher[22], and smoke and alcohol history has a positive correlation with the increasing incidence of complications[23], such as the higher incidence of infection, flap failure, deep vein thrombosis(DVT), delayed union and nonunion which always signifies worse prognosis and higher hospital cost[24]. There were 2 cases with postoperative surgical site infection in our study accepting additional debridement surgery and changing of implants, 3 cases was transferred to department of internal medicine for treatment of lung infection, 2 cases of the bedsore, 13 cases with onset of DVT and 7 of them were received inferior vena cava filter implantation to decrease the risk of pulmonary embolism because of the diameter of thrombosis were over $8 \mathrm{~mm}, 2$ case with delayed union finally gained radiographic union after performing the bone grafting. On the other hand, the postoperative rehabilitation 
or follow-up expenses depend on the financial condition of the family, the compliance of the patient and the emphasis for the fracture healing.

There are also some lacks of our study which should be clarified. The sample size of our retrospective study was relatively small that may enhance the probability of bias to the statistical results. Thus further prospective studies with larger samples are required to validate our findings. Also we investigated the total cost within just 1 year after surgery between the two groups, the time span could be longer since a longer rehabilitation and follow-up indicated a more precise clinical outcomes and cost results for type 43-A open fractures.

\section{Conclusion}

The implants fixation decision making is always a substantial challenge for clinical orthopedic surgeon to increase the clinical outcomes and decrease the financial burden for patients. Here we consider both the IMN and LCP could provide a satisfactory outcome for open distal tibial fractures. However, the IMN was a more cost-effective management than LCP when the economic analysis was included.

\section{Abbreviation}

IMN: intramedullary nailing, LCP: locking compression plate, CNY: Chinese Yuan, MESS: mangled extremity severity score, CER: cost-effectiveness ratio, ICER: incremental cost-effectiveness ratio, DVT: deep vein thrombosis.

\section{Declarations}

\section{Ethics approval and consent to participate}

This study was performed in accordance with the Declaration of Helsinki as revised in 2008 and was authorized by the Ethics Committee of Xi'an Honghui Hospital.

\section{Availability of data and materials}

The data and materials are available from the medical records department of Xi'an Honghui Hospital.

\section{Competing interests}

Yibo Xu, Cheng Ren, Teng Ma, Deyin Liu, Yao Lu, Ming Li, Hongliang Liu, Wang Tao, Ning Duan, Ning Jiao, Daigang Lu, Qian Wang, Hanzhong Xue, Zhong Li and Kun Zhang declare that they have no conflict of interest.

\section{Funding}

Not applicable 
Author's contributions

ZL and KZ Designed the study, modified and approved the final version of the manuscript. YBX and CR wrote the first draft of the manuscript. TM DYL YL ML HLL WT ND NJ DGL QW and HZX collected, analyzed and interpreted the data. All authors read and approved the final manuscript.

\section{References}

1. Zalavras CG, Patzakis MJ. Open fractures: evaluation and management. J Am Acad Orthop Surg. 2003;11(3):212-9.

2. Gustilo RB, Merkow RL, Templeman D. The management of open fractures. J Bone Joint Surg Am. 1990;72(2):299-304.

3. Yu B. Current situation and development of treatment of open fractures. Chin J Orthop Trauma, 2019,21(10): 835-8. doi:10.3760/cma.j.issn.1671-7600.2019.10.002.

4. Court-Brown CM, Bugler KE, Clement ND, Duckworth AD, McQueen MM. The epidemiology of open fractures in adults. A 15-year review. Injury. 2012;43(6):891-7.

5. Weber CD, Hildebrand F, Kobbe P, Lefering R, Sellei RM, Pape HC. Epidemiology of open tibia fractures in a population-based database: update on current risk factors and clinical implications. Eur $\mathrm{J}$ Trauma Emerg Surg. 2019;45(3):445-53.

6. Young K, Aquilina A, Chesser TJS, Costa ML, Hettiaratchy S, Kelly MB et al. Open tibial fractures in major trauma centres: A national prospective cohort study of current practice. Injury. 2019;50(2):497502.

7. Johner $\mathrm{R}$, Wruhs 0 . Classification of tibial shaft fractures and correlation with results after rigid internal fixation. Clin Orthop Relat Res. 1983(178):7-25.

8. Anglen JO. Wound irrigation in musculoskeletal injury. J Am Acad Orthop Surg. 2001;9(4):219-26.

9. Southam BR, Archdeacon MT. "latrogenic" Segmental Defect: How I Debride High-Energy Open Tibial Fractures. J Orthop Trauma. 2017;31 Suppl 5:S9-s15.

10. Ma T, Wang Q, Lu Y, Sun L, Lu DG, Li M, et al. Rapid judgment of distal inward or outward rotation during closed reduction and intramedullary nailing for complex tibial fractures[J]. Chin J Orthop Trauma, 2017,19(10): 874-9.

11. Olson SA. Open fractures of the tibial shaft. Instr Course Lect. 1997;46:293-302.

12. Rymer B, Dimovska EOF, Chou DTS, Choa R, Davis B, Huq S. A representative assessment of the management of open fractures of the lower limb within UK orthoplastic centres: A two-centre audit of compliance with national standards. Injury. 2017;48(10):2266-9.

13. Halawi MJ, Morwood MP. Acute Management of Open Fractures: An Evidence-Based Review. Orthopedics. 2015;38(11):e1025-33.

14. Neumann PJ, Sanders GD. Cost-Effectiveness Analysis 2.0. N Engl J Med. 2017;376(3):203-5. 
15. Federer AE, Mather RC, 3rd, Ramsey ML, Garrigues GE. Cost-effectiveness analysis of total elbow arthroplasty versus open reduction-internal fixation for distal humeral fractures. J Shoulder Elbow Surg. 2019;28(1):102-11.

16. Hu L, Xiong Y, Mi B, Panayi AC, Zhou W, Liu Y et al. Comparison of intramedullary nailing and plate fixation in distal tibial fractures with metaphyseal damage: a meta-analysis of randomized controlled trials. J Orthop Surg Res. 2019;14(1):30.

17. Wang B, Zhao Y, Wang Q, Hu B, Sun L, Ren C et al. Minimally invasive percutaneous plate osteosynthesis versus intramedullary nail fixation for distal tibial fractures: a systematic review and meta-analysis. J Orthop Surg Res. 2019;14(1):456.

18. Briel M, Sprague S, Heels-Ansdell D, Guyatt G, Bhandari M, Blackhouse $G$ et al. Economic evaluation of reamed versus unreamed intramedullary nailing in patients with closed and open tibial fractures: results from the study to prospectively evaluate reamed intramedullary nails in patients with tibial fractures (SPRINT). Value Health. 2011;14(4):450-7.

19. Haonga BT, Areu MMM, Challa ST, Liu MB, Elieza E, Morshed S et al. Early treatment of open diaphyseal tibia fracture with intramedullary nail versus external fixator in Tanzania: Cost effectiveness analysis using preliminary data from Muhimbili Orthopaedic Institute. Sicot-j. 2019;5:20.

20. Galal S. Minimally invasive plate osteosynthesis has equal safety to reamed intramedullary nails in treating Gustilo-Anderson type I, II and III-A open tibial shaft fractures. Injury. 2018;49(4):866-70.

21. Wang JQ, Chen ZX, Guo WJ, Zhao YM, Peng L. Comparison of plate and intramedullary nail fixation of extra-articular tibial fractures: A retrospective study exploring hidden blood loss. Injury. 2019;50(2):546-50.

22. Li Z, Chang X, Wang YJ, Fan Y, Gao P, Zhang J, et al. Statistical analysis on hospitalization expenses of patients with osteoporotic fracture undergoing surgery. Chin $\mathrm{J}$ Bone and Joint Surgery,2019,12(10):786-790.

23. Adams $\mathrm{Cl}$, Keating JF, Court-Brown CM. Cigarette smoking and open tibial fractures. Injury. 2001;32(1):61-5.

24. 24. Parker B, Petrou S, Masters JPM, Achana F, Costa ML. Economic outcomes associated with deep surgical site infection in patients with an open fracture of the lower limb. Bone Joint J. 2018;100b(11):1506-10.

\section{Tables}


Table 1: The demographic data between the two groups.

\begin{tabular}{|llll|}
\hline Characteristics & Group IMN & Group LCP & $\mathrm{p}$ \\
\hline Number of patients & 28 & 21 & \\
\hline Gender(male/female) & $24 / 4$ & $17 / 4$ & 0.655 \\
\hline Age(year) & $49.0 \pm 10.6$ & $53.4 \pm 14.7$ & 0.698 \\
\hline History of smoke and alcohol(yes/no) & $21 / 7$ & $13 / 8$ & 0.325 \\
\hline Comorbidity disease(yes/no) & $7 / 21$ & $5 / 16$ & 0.924 \\
\hline Injury mechanism & & & \\
\hline Traffic accident & 18 & 9 & 0.136 \\
\hline Other & 10 & 12 & \\
\hline AO classification & & & 0.801 \\
\hline Type 43-A1 & 3 & 2 & \\
\hline Type 43-A2 & 12 & 11 & \\
\hline Type 43-A3 & 13 & 8 & 0.137 \\
\hline Gustilo classification & & & \\
\hline Type I & 1 & 4 & \\
\hline Type II & 12 & 10 & \\
\hline Type III & 15 & 7 & \\
\hline Mess & $5.43 \pm 0.79$ & $5.07 \pm 0.92$ & 0.390 \\
\hline
\end{tabular}


Table 2: Comparison of Injury to surgery interval, times of debridement surgery, surgical duration, intraoperative bleeding loss, length of hospital stay, complications, excellent and good rate, average costs between the two groups.

\begin{tabular}{|llll|}
\hline Characteristics & Group IMN & Group LCP & $\mathrm{p}$ \\
\hline Injury to surgery interval(h) & $11.86 \pm 6.59$ & $11.43 \pm 3.69$ & 0.849 \\
\hline Times of surgical debridement & $1.57 \pm 0.79$ & $2.00 \pm 1.04$ & 0.350 \\
\hline Total surgical duration(min) & $247.50 \pm 57.94$ & $350.00 \pm 178.77$ & 0.017 \\
\hline Intraoperative bleeding loss $(\mathrm{ml})$ & $243.18 \pm 118.82$ & $467.86 \pm 490.53$ & 0.046 \\
\hline Total length of hospital stay $(\mathrm{d})$ & $14.3 \pm 6.3$ & $15.9 \pm 4.7$ & 0.625 \\
\hline Complications & $13(46.4 \%)$ & $9(42.9 \%)$ & 0.804 \\
\hline Excellent and good rate & $23(82.1 \%)$ & $18(85.7 \%)$ & 0.738 \\
\hline Average costs $(\mathrm{CNY})$ & $126435.90 \pm 39093.98$ & $147834.60 \pm 56821.12$ & 0.048 \\
\hline
\end{tabular}

\section{Figures}



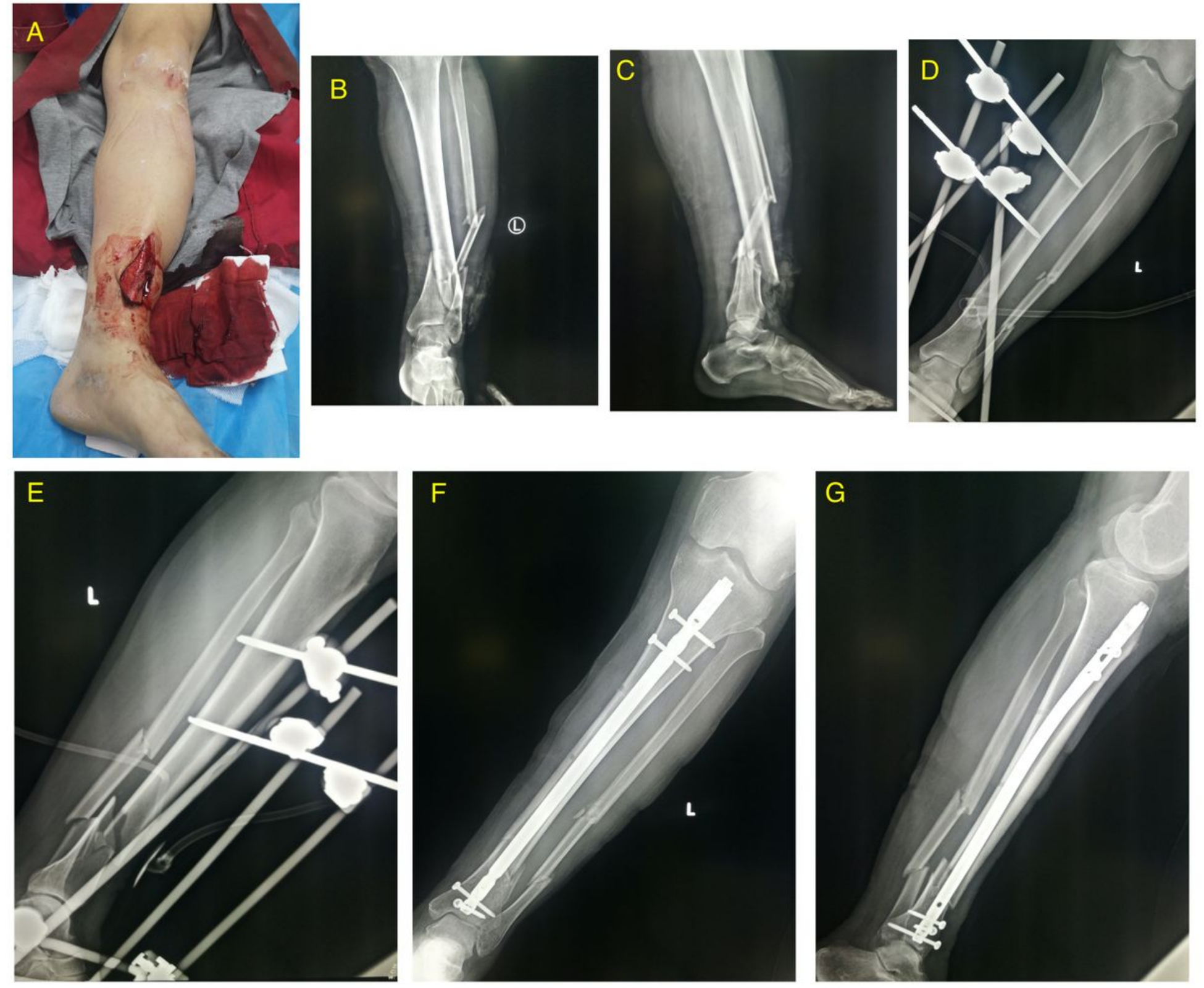

\section{Figure 1}

The IMN fixation for open distal tibial fracture. The patient is a 47-year-old male, traffic accident injury causing left calf pain and limited activity. a wound appearance, b.c preoperative AP and lateral X-ray, d.e AP and lateral X-ray after temporary external fixation between debridement surgeries, f.g AP and lateral Xray after intramedullary nailing fixation. 

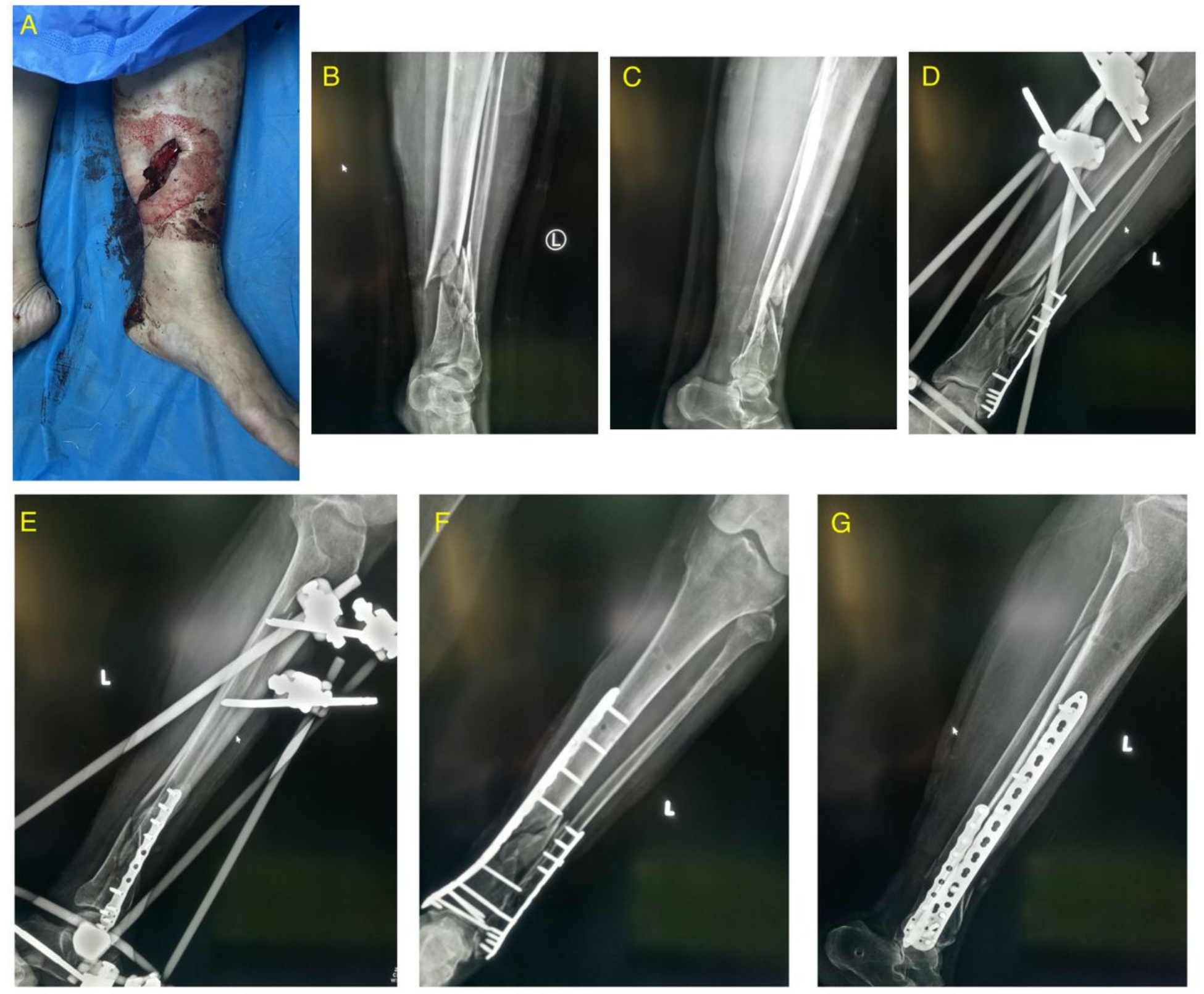

\section{Figure 2}

The LCP fixation for open distal tibial fracture. The patient is a 61-year-old female, traffic accident injury causing left calf pain and limited activity, a wound appearance, b.c preoperative AP and lateral X-ray, d.e AP and lateral X-ray after temporary external fixation between debridement surgeries, f.g AP and lateral Xray after plate and screws fixation. 